\title{
A migração ambiental no regime internacional de mudança climática sob a perspectiva da AOSIS
}

\author{
The environmental migration in the international climate change \\ regime from the perspective of AOSIS
}

Thales Jéferson Rodrigues Schimitt Verônica Korber Gonçalves²

\begin{abstract}
Resumo: a migração ambiental tem ganhado destaque, nas últimas décadas, como a dimensão humana dos impactos da mudança climática e como efetiva estratégia de adaptação para comunidades em regiões vulneráveis. Este artigo analisa como a migração ambiental é apresentada no discurso da Aliança dos Pequenos Estados Insulares (AOSIS), grupo de negociação dos países mais afetados pelo aquecimento global, no regime internacional de mudança climática. Através da análise de fontes primárias, constata-se que o processo migratório é utilizado como legitimador moral das propostas de mitigação da AOSIS, sem ser considerado como proposta de adaptação. Diante de aparente incoerência entre o uso da migração no discurso e a ausência de medidas efetivas de adaptação, apresenta-se possíveis explicações sob quatro aspectos: político, cultural, técnico e estrutural
\end{abstract}

Palavras-chave: Migração ambiental; AOSIS; Adaptação; Regime internacional de mudança climáticas

\begin{abstract}
: environmental migration has gained the spotlight over recent decades both as the human dimension of climate change impacts and as an effective adaptation strategy for communities in vulnerable regions. This paper analyzes how environmental migration is presented in the discourse of the Alliance of Small Island States (AOSIS), the negotiation group of countries most affected by global warming, in the climate change regime. Through analysis of primary sources, it is noted that the migration process is used as a moral legitimation of AOSIS' mitigation proposals, without being considered as an adaptation proposal. Before this apparent inconsistency between the use of migration in the discourse and the lack of adaptation effective measures, possible explanations are presented regarding political, cultural, technical and structural aspects.
\end{abstract}

Keywords: Environmental migration; AOSIS; Adaptation; Climate change regime

\section{Introdução}

Com o que há de mais recente no conhecimento científico relativo à mudança climática, projeta-se impactos consideráveis até o fim do século XXI, especialmente em ecossistemas relacionados às dinâmicas oceânicas. Fenômenos de salinização, erosão e enchentes já são observados 
como consequência do aumento do nível do mar, deixando comunidades costeiras em situação de vulnerabilidade. Em 2100, estima-se que entre 31 e 69 milhões de pessoas estarão expostas a enchentes, aumentando fluxos de deslocamento e realocação, e isso em um cenário de $1,5^{\circ} \mathrm{C}$ de aquecimento acima dos níveis pré-industriais, considerado o resultado ideal do Acordo de Paris (HOEGH-GULDBERG et al., 2018). Ademais, as metas de redução de emissão de gases de efeito estufa das contribuições nacionalmente determinadas de 2015 implicam em um aquecimento entre 2,6 e $3,1^{\circ} \mathrm{C}$, que aumenta exponencialmente os impactos da mudança climática (ROGELJ et al., 2016).

Entre os principais afetados estão as populações dos Estados insulares, que estão em situação de vulnerabilidade devido a desafios de desenvolvimento que não decorrem somente da baixa altitude (KELMAN, 2014). Países como Kiribati e Tuvalu já observam degradação da terra por intrusão de água marinha, enquanto a Polinésia Francesa passa por mudanças da linha costeira. Tais comunidades apresentam alta sensibilidade às mudanças em ecossistemas costeiros, como o aquecimento do oceano, a maior frequência de ciclones e o aumento do nível do mar, que degrada a terra e afeta a disponibilidade de recursos hídricos potáveis. A alta mortalidade de corais, que são fator importante para subsistência de comunidades insulares, também se apresenta como um forte impacto negativo - projeta-se que entre 70 e $90 \%$ dos recifes de corais ao redor do mundo fiquem em risco de degradação a longo prazo com aquecimento de $1,5^{\circ} \mathrm{C}$; a $2^{\circ} \mathrm{C}$, a estimativa sobe para $99 \%$. As ameaças relacionadas ao aumento do nível do mar permanecerão para os Estados insulares em desenvolvimento após o século $\mathrm{XXI}$, mesmo com aquecimento menor que $1,5^{\circ} \mathrm{C}$, e entre os impactos mais nocivos está a projetada inabitabilidade de diversos atóis, que pode modificar profundamente a estrutura das sociedades estabelecidas nas ilhas (HOEGH-GULDBERG et al., 2018). Tais comunidades insulares são representadas internacionalmente pela Aliança dos Pequenos Estados Insulares (AOSIS, sigla em inglês para Alliance of Small Island States), que abrange 44 países e aproximadamente $5 \%$ da população mundial.

A migração' relaciona-se às mudanças climáticas tanto como consequência de seus impactos, quanto como medida de adaptação observada. Consequência, pois, o deslocamento humano é mandatório em situações de desastre e perda de lares cuja origem é a mudança do clima, e medida de adaptação pois também é uma estratégia para minimizar os danos causados por impactos ambientais. Apresenta riscos próprios e vulnerabilidades emergentes decorrentes da possibilidade de disrupção social, mas também pode aprimorar capacidades de resiliência de novas comunidades formadas no local de destino dos migrantes a partir do compartilhamento de experiências (CORENDEA; MANI, 2018; HOEGH-GULDBERG et al., 2018).

Nesse contexto, o objetivo deste artigo é analisar como se estabelece a discussão da migração induzida pelas mudanças climáticas e por seus impactos ambientais dentro do regime internacional de mudança climática. Mais especificamente, busca-se compreender a atuação da AOSIS. A Aliança participa ativamente das negociações internacionais sobre clima e meio ambiente como porta-voz de seus Estados-membros, apresentando os interesses das nações mais prejudicadas com as mudanças climáticas. Para isso, recorre-se à análise do conteúdo do discurso da Aliança em documentos das Conferências das Partes (COPs, sigla em inglês para Conference of Parties) da Convenção-Quadro das Nações Unidas sobre Mudança do Clima, que, desde 1992, é o principal fórum do regime internacional de mudança climática. 
A próxima seção introduz as escolhas metodológicas para a análise feita neste trabalho. As duas seções posteriores apresentam o contexto da discussão; primeiramente, uma revisão de literatura sobre migração ambiental como consequência da mudança climática e de estratégia de adaptação, e após, um olhar sobre a atuação da AOSIS e suas especificidades no regime internacional de mudança climática. A quinta seção envolve os resultados da análise documental, com posterior discussão dos achados, considerando o contexto dos Estados insulares. Pretende-se, por fim, avaliar quais são as possibilidades que se identificam no regime internacional de mudança climática para abordar a migração ambiental em instrumentos multilaterais sob a perspectiva da AOSIS.

\section{Metodologia}

O objetivo da análise é conhecer elementos do discurso da AOSIS em relação à migração ambiental, por se reconhecer que os discursos têm um papel importante na construção da política externa e dos resultados documentais das negociações (LARSEN, 1997). Para isso, recorre-se a fontes primárias, a saber, documentos oficiais de representações da AOSIS. Busca-se reconstituir o sentido dado para a migração por parte da Aliança especificamente na discussão internacional que abrange a mudança climática. Ao todo, foram analisados 84 documentos disponibilizados online pela AOSIS, além da revisão bibliográfica e fontes secundárias. Tais documentos são pronunciamentos oficiais nas COPs e submissões a fóruns subsidiários no regime internacional de mudança climática, em última instância ligados à Convenção-Quadro das Nações Unidas sobre Mudança do Clima, em que se incluem o Órgão Subsidiário de Assessoramento Científico e Tecnológico, o Órgão Subsidiário de Implementação, a Plataforma de Durban e outros grupos de trabalho ad hoc referentes ao tema. Em casos específicos de relevância, também utilizou-se publicações pontuais da Aliança, como a Declaração de Líderes da AOSIS, por estipular diretrizes gerais para a política externa do grupo. A escolha de analisar principalmente documentos de COPs e estritamente relacionados deve-se à centralidade das Conferências no regime internacional de mudança climática. A COP é a reunião internacional anual de maior importância para o regime, envolvendo não somente representantes de Estados, mas também forte cobertura midiática e envolvimento da sociedade civil. Os documentos que surgem das Conferências estabelecem as propostas internacionais para lidar com a mudança do clima. O recorte temporal da pesquisa enfoca os anos de 2012 a 2018. O período escolhido para análise justifica-se por abarcar o momento de gestação e introdução do Acordo de Paris, estabelecido em 2015, que passa a ser o documento central para o regime internacional do clima.

A análise do conteúdo do discurso foi realizada em torno de cinco termos-chave: "migration" (migração), "refugee" (refugiado), "displacement" (deslocamento), "relocation" (relocação) e "resettlement" (reassentamento). Esses termos abrangem praticamente todas as formas de movimento humano. Os dois primeiros são categorias delimitadas juridicamente e tratadas nas Relações Internacionais; os demais termos, apesar de poderem ser ambíguos em determinados contextos, são utilizados diversas vezes referindo-se a processos de natureza migratória, pelo que é necessário incluí-los na análise. A partir da busca de tais termos, procurou-se compreender o seu uso pela AOSIS, observando o documento de origem (Qual a discussão central do documento? É 
delimitado a propostas específicas ou é mais abrangente?) e a construção do argumento em que o termo se encontra (Qual o papel do termo? Aparece como proposta efetiva de ação - planejamento migratório, por exemplo - ou é justificativa para outra proposta?). Ao final, buscou-se encontrar os principais empregos que eram dados aos termos escolhidos e identificar a possível preponderância de algum uso específico. Assim, chegou-se a como a AOSIS utilizou o processo migratório nas discussões no regime internacional de mudança climática.

\section{Migração ambiental: entre o impacto da mudança climática e a adaptação}

O termo "migração ambiental", assim como as variantes "migração climática", "refugiado ambiental" e "refugiado climático", envolve extenso debate no meio acadêmico e político. Diversas tentativas de categorização já foram propostas, buscando o reconhecimento para garantir proteção e assistência aos migrantes que se deslocam por causa de alterações no ambiente que afetam negativamente suas condições de vida. Atualmente, migrantes ambientais permanecem em um vácuo jurídico, por não se enquadrarem na definição prevista na Convenção relativa ao Estatuto dos Refugiados de 1951, tratado internacional multilateral que aborda especificamente a situação de refúgio, já que não há fundado temor de perseguição dentro das causas preestabelecidas - raça, religião, grupo social, etnia ou opinião política (CORENDEA, 2016). Quanto à migração em si, não há um tratado internacional multilateral que estabeleça compromissos legalmente vinculantes abordando a questão. $\mathrm{O}$ último avanço nas negociações internacionais no tema resultou no Pacto Global para a Migração Segura, Ordenada e Regular, assinado em 19 de dezembro de 2018 em Nova lorque, que reconhece a influência da degradação ambiental e dos efeitos adversos das mudanças climáticas no processo migratório (GLOBAL, 2018), entretanto sua efetividade depende de implementação por parte dos Estados que se comprometeram com o documento, entre os quais não estão países relevantes como Estados Unidos e Brasil (ONU, 2019). Mesmo com a Convenção de 1951 e com o Pacto Global para Migração, não há consenso político ou jurídico sobre a definição de migração ambiental.

O termo "migração ambiental" pode ser definido como o fluxo migratório, seja atravessando fronteiras nacionais ou não, relacionado a processos de degradação da qualidade de vida decorrentes direta ou indiretamente de impactos ambientais, não somente naturais, entre os quais os provenientes da mudança do clima. Os limites do que constitui um fluxo migratório majoritariamente causado por fatores ambientais (especialmente em caso de impactos de longo prazo, como aumento do nível do mar) ainda não são bem definidos, tendo em consideração que a migração é um fenômeno complexo e multifacetado, apresentando diversas causas e motivações distintas (MORTREUX; BARNETT, 2009; KELMAN, 2014). No fim dos anos 1990 e início dos anos 2000, surgiu destacada preocupação com a migração ambiental como um "fenômeno crescente" e diversos cálculos foram feitos com relação ao número de pessoas em tal situação. Segundo Myers (2002, p. 610), uma estimativa de 25 milhões de" refugiados ambientais" em 1995 seria ainda "cuidadosa e conservadora", e tal número provavelmente dobraria até 2010. Contudo, nos anos posteriores, as tentativas de estimação foram objeto de críticas, especialmente por falta de metodologia definida 
e fontes confiáveis para as aferições (GEMENNE, 2011). Adiciona-se ao ceticismo com relação à migração ambiental o fato de que as mudanças climáticas são somente um dos fatores da decisão por migrar, sendo que o fenômeno migratório é parte constitutiva da vida diária nas comunidades insulares mais afetadas e é caracterizado por motivações coletivas, especialmente no Pacífico (FARBOTKO; LAZRUS, 2012). Ainda assim, mesmo autores mais céticos reconhecem que a migração ambiental é um tópico relevante para os Estados insulares, principalmente para o futuro, seja como consequência das mudanças climáticas ou como medida de adaptação (KELMAN, 2014).

As estimativas de impactos futuros das mudanças climáticas, associadas ao desgaste das negociações ambientais internacionais nos últimos anos, apontam a necessidade de se construir possibilidades para a migração ambiental que alterem menos as estruturas das comunidades afetadas, já que se projeta o aumento da migração relacionada à mudança climática, bem como a possibilidade de populações tropicais terem que deslocar-se mais que 1000 quilômetros devido a desastres naturais, gerando brusco aumento de densidade populacional em áreas pontuais (HOEGH-GULDBERG et al., 2018).

Como consequência das mudanças climáticas, a migração tende a erodir a resiliência e aumentar as vulnerabilidades, na medida em que laços culturais com o território significativos para as comunidades afetadas são indesejavelmente perdidos (ADGER et al., 2013). Entretanto, a migração como medida de adaptação planejada e eficiente pode resultar em condições melhores para os migrantes. A adaptação como estratégia para lidar com as mudanças climáticas é o "ajuste em sistemas naturais e/ou humanos em resposta a estímulos climáticos atuais ou esperados ou seus efeitos" (MINISTRY OF ECONOMY OF REPUBLIC OF FIJI, 2018, p. 6), buscando reduzir impactos e, se possível, transformar impactos negativos em positivos (TOL, 2005). Distingue-se da estratégia de mitigação na medida em que esta busca evitar previamente o próprio fenômeno que gera os impactos ambientais, de que é exemplo a política de redução de emissões de gases de efeito estufa. Enquanto a mitigação é tipicamente estudada no campo da política internacional e da macroeconomia, gerando medidas de implementação de larga escala, a adaptação é normalmente de natureza local, sendo abordada sob perspectivas microeconômicas (BOSELLO; CARRARO; DE CIAN, 2010). A relação mitigação-adaptação tem dominado as discussões internacionais sobre mudanças climáticas nas últimas décadas e, apesar de serem estratégias complementares, permanecem separadas por abordagens fundamentalmente distintas para solucionar os mesmos problemas ambientais (BIESBROEK; SWART; VAN DER KNAAP, 2009).

Segundo Thomas e Benjamin (2018), a migração planejada de comunidades que se encontram em regiões vulneráveis é uma estratégia de adaptação particularmente útil para Estados insulares, na medida em que confrontam perdas e danos. Em comparação com processos migratórios ad hoc, a migração planejada resulta em menor vulnerabilidade, todavia, deve ser estruturada com participação ativa da própria comunidade migrante, visando a diminuição da possibilidade de disrupção social (THOMAS; BENJAMIN, 2018).

Ademais, a migração apresenta a propensão a aumentar a resiliência transformativa tanto das comunidades migrantes quanto das nativas receptoras, na medida em que a capacidade de adaptação do sistema socio ecológico melhora com as experiências compartilhadas (CORENDEA; MANI, 2018). A reconstrução de comunidades oferece a possibilidade de evitar vulnerabilidades 
semelhantes às anteriores, abordando múltiplos desafios de desenvolvimento que ofereciam risco para além da mudança climática (KELMAN, 2014).

A migração ambiental não é tema ignorado no cenário político internacional, e tem aparecido com frequência - e até mesmo de forma exagerada - na mídia, que ressalta o impacto humano da mudança climática (MCNAMARA; GIBSON, 2009). Ainda assim, as alternativas práticas são controversas e sempre consideradas como último recurso.

\section{AOSIS no regime internacional de mudança climática}

A AOSIS tem se colocado ativamente no regime internacional de mudança climática como um grupo de negociação desde 1990, com o propósito de unir os países em vulnerabilidade e fazer seus interesses serem ouvidos (WONG, 2011). Seu surgimento se dá no contexto da publicação do Primeiro Relatório do Painel Intergovernamental sobre Mudanças Climáticas (IPCC, sigla em inglês para Intergovernmenta I Panel on Climate Change), que ressaltou o impacto das mudanças climáticas especificamente em ilhas.

Pequenos Estados insulares carecem de poder estrutural e material para influenciar significativamente as negociações internacionais. O processo de formação da AOSIS, portanto, constitui-se em um agrupamento contra a marginalização de tais países no sistema internacional, de forma que tais Estados possam exercer um papel relevante no regime de mudança climática, que está diretamente relacionado a seus interesses (BHANDARY, 2017). Para compreender a atuação da AOSIS nesse contexto, Betzold (2010) destaca que a Aliança, na falta de fontes tradicionais de poder, utiliza-se de "poder emprestado" para influenciar as negociações internacionais. Entre as estratégias do grupo está o uso de fontes externas de poder, como ONGs, que ajudaram a traduzir informação científica em posição política, e o apelo a normas e princípios. Ainda que a AOSIS não tenha sido bem sucedida em criar os resultados desejáveis no curto prazo, seu uso de "poder emprestado" possibilitou uma influência desproporcional nos processos de negociação (BETZOLD, 2010). É interessante observar, entretanto, que ao longo dos anos algumas das insistências da Aliança desde as primeiras negociações aparecem também como resultado, como é o caso da limitação do aumento da temperatura global a $1,5^{\circ} \mathrm{C}$ acima dos níveis pré-industriais, que aparece no texto do Acordo de Paris, ainda que como modesta aspiração (FALKNER, 2016).

Particularmente elucidativo é o estudo realizado por de Águeda Corneloup e Mol (2014), que considera a atuação da AOSIS dentro das estratégias de liderança alternativas propostas no modelo da "barganha institucional". Nesse quadro teórico, há quatro estratégias de liderança utilizadas em regimes: liderança estrutural, que traduz recursos materiais em poder; liderança empreendedora, que se refere à capacidade de influenciar a maneira como questões são apresentadas na negociação através de iniciativas diplomáticas proativas; liderança intelectual, que traduz conhecimento científico em argumento político (YOUNG, 1991); e liderança direcional (também chamada de ambiental por de Águeda Corneloup e Mol), que se baseia em incentivar determinadas propostas de soluções pelo exemplo de adotá-las na prática (SKODVIN; ANDRESEN, 2006).

Para a AOSIS, a liderança estrutural é pouco relevante, pois suas capacidades materiais são limitadas; portanto, a Aliança faz uso de todas as demais estratégias de liderança para conseguir influen- 
ciar nas negociações, promovendo campanhas junto à sociedade civil - como a "1.5 to Stay Alive" -, realizando parcerias com ONGs e cientistas, e buscando a renovação da matriz energética para alternativas renováveis por diversos Estados-membros (DE ÁGUEDA CORNELOUP; MOL, 2014).

Além das estratégias de liderança já estabelecidas na literatura, de Águeda Corneloup e Mol apontam um fator de especial relevância utilizado pela AOSIS nas negociações no regime de mudança climática: a moral. Na busca de legitimidade e normatividade no tocante à questão ambiental, a moralidade surge como uma nova estratégia relativamente independente. Essa "liderança" moral é particularmente bem empregada pela Aliança, embora seja identificada uma tendência mais ampla de seu uso por outros atores, especialmente no regime de mudança climática (DE ÁGUEDA CORNELOUP; MOL, 2014).

Desde 1990, a AOSIS tem apresentado uma atuação estável, não sendo observada grande variação nas estratégias utilizadas nas negociações (BETZOLD, 2010). A Aliança permanece ainda como um grupo coordenado e coeso, especialmente em relação às questões de mitigação e adaptação, sendo ator central no regime internacional de mudança climática (BETZOLD; CASTRO; WEILER, 2012). Assim, podemos analisar a atuação da AOSIS na discussão da migração ambiental com base nas estratégias usualmente utilizadas em outras discussões e identificadas na literatura.

\section{Migração ambiental no discurso da AOSIS}

Após a breve contextualização acerca da migração ambiental e dos impactos das mudanças climáticas para os países da AOSIS, apresenta-se, agora, de que forma a Aliança refere-se à migração ambiental em sua atuação no regime internacional de mudança climática.

Primeiramente, é preciso destacar que a migração ambiental não é um tema ignorado pela AOSIS ou imposto pela agenda de outros atores. Antes, apresenta-se como uma das principais preocupações da Aliança reconhecida na Declaração de Líderes do grupo (AOSIS, 2012a). Dos vinte pontos levantados na declaração, dois tratam diretamente de deslocamento e realocação. O ponto 3 ressalta a ocorrência de migração forçada devido aos impactos das mudanças climáticas e a possibilidade de ilhas tornarem-se inabitáveis ou submergirem "causando deslocamento em massa devido à mudança climática"; o ponto 4 coloca o deslocamento forçado e a realocação de comunidades como "implicações securitárias da mudança climática" que exigem consideração urgente (AOSIS, 2012a, p. 2).

É possível identificar dois momentos distintos na análise: de 2012 a 2015 as negociações estavam centradas em criar um novo acordo que lidasse com a questão da mudança do clima de forma mais efetiva que o antigo Protocolo de Quioto e que conseguisse ampla adesão internacional; após a COP21, em 2015, com o Acordo de Paris formulado, as discussões passam a enfocar a implementação do acordo. Nesse sentido, encontra-se no período 2012-2015 uma maior abertura para propostas referentes à mudança climática, havendo possibilidade de tratar da migração ambiental (especialmente em mecanismos que lidassem com perdas e danos, como a AOSIS chegou a explicitar), contrastando tanto com os anos anteriores quanto posteriores. No período 2015-2018, embora a liberdade de propostas diminua, discute-se as possibilidades de implementação do Acordo de Paris, especialmente relevante para compreender o status da migração ambiental atualmente no regime internacional de mudança climática. 
A principal (e virtualmente única) perspectiva em que é apresentada a migração ambiental nos documentos analisados é a de consequência das mudanças climáticas, de dimensão humana do aquecimento global. Neste sentido, somente relata-se a migração, porém não se propõe abordagem específica para o problema e nem se abre espaço para discutir efetivamente os sujeitos do processo migratório e futuras ações. Chega-se mesmo a citar eventos climáticos específicos e seus impactos:

Infelizmente, sabemos que impactos climáticos severos já estão ocorrendo e que muitos piores ainda estão por vir. Somente na semana passada, as Ilhas Marshall e Kiribati foram severamente danificadas pelas chamadas 'King Tides' [as marés mais altas] e milhares de pessoas tiveram que ser realocadas internamente. (AOSIS, 2014, p. 3) ${ }^{3}$

Por outro lado, a migração entendida como estratégia de adaptação permanece ausente no discurso da AOSIS e não há menção ao desenvolvimento de qualquer iniciativa de migração planejada. A única exceção encontrada é a possibilidade aberta pela discussão sobre a implementação de um mecanismo relativo a perdas e danos na gestação do Acordo de Paris, entretanto as ligações entre migração e perdas e danos ainda são minoria e o assunto é relativamente novo (THOMAS; BENJAMIN, 2018).

Ademais, medidas de adaptação são discutidas pela Aliança com enfoque no funcionamento de fundos e na necessidade de apoio financeiro externo para lidar com os efeitos da mudança climática. A preocupação maior do grupo é nitidamente o aumento da ambição de mitigação, que é apresentada como antecedente a qualquer negociação sobre adaptação no estabelecimento de protocolos:

Será difícil proceder muito adiante em nossas discussões sobre o novo Protocolo [o futuro Acordo de Paris] até sabermos que a lacuna da mitigação pré-2020 será preenchida. [...] Profundas implicações para o que a AOSIS irá exigir no novo acordo decorrem de preenchermos ou não a lacuna. Por exemplo, podemos confiar no Comitê de Adaptação para realocar completamente toda a nossa infraestrutura crítica mais para o interior? O Fundo Verde do Clima será capaz de pagar por tudo isso? (AOSIS, 2012b, p. 1-2)4.

De fato, a maior parte das referências à migração, deslocamento e realocação de comunidades se dá em contexto de discussão de medidas de mitigação. Neste sentido, o processo migratório é tão somente parte de um argumento voltado para a necessidade de reduzir emissões de gases de efeito estufa. Não há no discurso preocupação real com os atuais migrantes e com abordar a situação de vulnerabilidade em que estão comunidades inteiras; antes, os sujeitos da migração tornam-se objetos na argumentação que contribuem para a defesa das propostas da Aliança referentes à mitigação. Constata-se, portanto, que a migração ambiental é utilizada pela AOSIS essencialmente como legitimador moral das suas propostas de mitigação. $O$ argumento abaixo serve de exemplo, apresentado como parte de uma defesa da

\footnotetext{
3 No original: "Unfortunately, we know that severe climate impacts are already ocurring and that much worse still lies ahead. Only last week the Marshall Islands and Kiribati were severely damaged by so-called 'King Tides' and thousands of people had to be internally relocated."

4 No original: "It will be difficult to proceed very far in our discussions on the new Protocol until we know that the pre-2020 mitigation gap will be closed. [...] Whether or not we close the gap will have profound implications for what AOSIS will require in the new agreement. For example, can we rely on the Adaptation Committee to completely relocate all of our critical infrastructure further inland? Will the Green Climate Fund be able to pay for it all?"
} 
necessidade de gerar políticas de mitigação guiadas pela ciência na implementação do novo Protocolo (que viria a ser o Acordo de Paris), que é legitimada pelos custos humanos:

Nós também possuímos uma melhor avaliação dos custos humanos da mudança climática, que estão aumentando rapidamente conforme ondas de calor, enchentes, aumento do nível dos mares, e outros impactos erodem meios de subsistência e deslocam comunidades em regiões vulneráveis ao redor do mundo. (AOSIS, 2012C, p.1)

Podem ser encontrados outros trechos semelhantes ao acima exposto que cumprem a mesma função de legitimação moral no discurso. O argumento da AOSIS é que, com a ocorrência de desastres climáticos que impactam severamente comunidades vulneráveis, transparece a necessidade urgente de combater as causas da mudança do clima, logo adotar medidas de mitigação torna-se não somente uma escolha política, mas o certo a se fazer diante dessa realidade.

Os resultados, portanto, apontam para uma aparente incoerência na discussão da migração ambiental estabelecida pela AOSIS. Embora o fenômeno migratório esteja diretamente relacionado com medidas de adaptação, sendo não somente uma estratégia possível, mas já presente em diversos casos, ele é majoritariamente utilizado pela Aliança relacionado a medidas de mitigação. A próxima seção busca discutir esse resultado trazendo o contexto dos Estados insulares e levantando possíveis motivações e causas que explicam o descompasso observado entre migração e adaptação.

\section{Discussão dos resultados}

A falta de relação entre a migração ambiental e as propostas de adaptação no discurso da AOSIS no regime internacional de mudança climática aparenta ser paradoxal. A migração, enquanto fenômeno atual e, em alguns casos, impossível de ser evitado, exige uma resposta pela via da adaptação. Assim, é natural esperar que os países da AOSIS, caracteristicamente sem poder estrutural relevante, promovam o debate sobre medidas de adaptação nas discussões internacionais, estando cientes da necessidade que os processos migratórios impõem. Entretanto, a análise do discurso da Aliança demonstra que a sua prioridade é a política de mitigação da mudança climática, tratando a migração somente como elemento moralmente legitimador das suas demais propostas.

Todavia, é possível identificar ao menos quatro motivações para a atuação da Aliança: política, cultural, técnica e estrutural.

A motivação política encontra sua expressão na resistência de embaixadores de ilhas do Pacífico em aceitar categorizações relativas à migração ambiental, como o estatuto de "refugiado climático". Esse posicionamento tem sido estável desde a fundação da AOSIS e baseia-se na defesa da soberania territorial do Estado, já que assumir a situação de refúgio significa "desistir" de seu território e colocar-se em condição passiva frente à mudança climática (MCNAMARA; GIBSON, 2009). Além disso, a construção de uma estrutura jurídica de amparo a migrantes ambientais é percebida em alguns Estados insulares como um prejuízo geopolítico, tendo em vista que isso poderia levar

\footnotetext{
5 No original: "We also possess a better appreciation of the human costs of climate change, which are mounting rapidly as heat waves, floods, rising seas, and other impacts erode livelihoods and displace communities in vulnerable regions around the world."
} 
países industrializados a deslocar seus esforços da redução de emissões de gases de efeito estufa para a realocação de comunidades afetadas (MCNAMARA; GIBSON, 2009; KOLMANNSKOG, 2012). Com essa prioridade dada às medidas de mitigação, a ausência de preocupação significativa com o fenômeno da migração ambiental acaba contribuindo para a abordagem ad hoc em casos em que o deslocamento é inevitável (THOMAS; BENJAMIN, 2018).

A motivação cultural expressa-se no desejo das populações insulares de permanecer em seu território. Residentes em ilhas, especialmente no Oceano Pacífico, têm uma forte ligação cultural com a terra, a tal ponto que "o povo, a terra e a cultura são mutuamente coexistentes e suportam continuamente um ao outro" (CORENDEA; MANI, 2018, p. 305). O senso de pertencimento ao lar ainda pode associar-se com a religião, como é o caso de Tuvalu, em que há o entendimento de que Deus fez Tuvalu para os tuvaluanos e que eles estão sob a proteção divina (MORTREUX; BARNETT, 2009). Ademais, povos insulares estão particularmente vulneráveis à perda de elementos materiais de sua cultura, provocando danos irreparáveis à memória geracional (ADGER et al., 2013). A concretização de fluxos migratórios, ainda que planejados, apresenta o grande desafio de cuidar para que a realocação afete o mínimo possível as estruturas características da sociedade e haja menor possibilidade de disrupção social; para isso, deve também considerar as perdas culturais, emocionais e psicológicas decorrentes da quebra do laço com a terra (CORENDEA; MANI, 2018; THOMAS; BENJAMIN, 2018). Como a cultura é «um instrumento para formação de decisão e implementação bem como o resultado final dessas políticas e das decisões implementadas», as políticas migratórias estatais estão sujeitas à sua influência, bem como são capazes de transformá-la radicalmente (KAVALIKU, 2005, p. 23).

A motivação técnica diz respeito à limitada capacidade administrativa, técnica e econômica dos Estados insulares, que inclusive é um dos motivos que levou à formação da AOSIS. A economia de diversas ilhas, principalmente no Pacífico, pode ser caracterizada, com algumas pequenas variações ao longo dos anos, pelo modelo MIRAB, que é um acrônimo para migração, remessas, ajuda e burocracia, em que transferências por conta corrente e produção não-comercializável são os setores que impulsionam o desenvolvimento econômico (BERTRAM, 1999). Outro setor escolhido por muitos países insulares é o do turismo internacional, que acarreta dependência de economias estrangeiras, especialmente da Europa e da América do Norte, bem como aumenta riscos provenientes de fatores exógenos (LEE; HAMPTON; JEYACHEYA, 2015). Essa inserção estruturalmente precária na economia internacional prejudica a capacidade dos Estados de responderem propriamente à mudança climática. Além disso, pequenos Estados insulares apresentam vulnerabilidades fundamentalmente políticas decorrentes de tal situação econômica que constituem desafios de desenvolvimento muito além do aquecimento global (KELMAN, 2014). Dessa forma, tais países ficam dependentes de ajuda externa para promover medidas de adaptação efetivas.

Por último, a motivação estrutural está ligada às instituições do regime internacional de mudança climática. A questão climática surgiu no final dos anos 1980 e estabeleceu-se formalmente no início dos anos 1990, com a criação de instituições como a Convenção-Quadro das Nações Unidas sobre Mudança do Clima e os protocolos posteriores. A grande influência ideológica desse período era o neoliberalismo, que preteria a ação coletiva em favor do estabelecimento de livres mercados internacionais, e que deixou sua marca no regime internacional de mudança climática (KLEIN, 2018). Como 
consequência, as negociações nas COPs focam em soluções globais, em detrimento de alternativas regionais, e baseadas em mecanismos de mercado, do que são exemplo os mercados de carbono. A migração é um tema considerado de baixa relevância no regime do clima por fatores diversos e complexos, entre os quais está a necessidade de abordagem local e não poder ser encaixado em qualquer mecanismo de mercado.

\section{Conclusão}

A migração ambiental apresenta-se como um tema pertinente para a AOSIS. Seus Estadosmembros apresentam alta vulnerabilidade aos impactos da mudança climática, logo o fluxo migratório subsequente precisa ser considerado em seus planejamentos políticos. De fato, alguns países já esboçam políticas migratórias nacionais como resposta à degradação do padrão de vida em suas terras, ainda que sejam modestas (CORENDEA; MANI, 2018; THOMAS; BENJAMIN, 2018).

Entretanto, a análise do conteúdo do discurso da AOSIS no regime internacional de mudança climática mostra que a migração ambiental é apresentada sob a perspectiva das consequências da mudança do clima essencialmente com o objetivo de legitimar propostas de mitigação através do apelo moral. Nesse sentido, o fenômeno atual da migração é desconsiderado nas pautas políticas dos Estados insulares para dar prioridade à luta pela redução das emissões de gases de efeito estufa, marcadamente buscando afirmar a responsabilidade das nações industrializadas quanto à degradação ambiental.

O foco em medidas de mitigação, embora aborde a raiz da questão climática e busque prevenir mais situações de vulnerabilidade que poderiam gerar processos migratórios, tem ocultado a necessidade de lidar com a migração ambiental já existente e que será inevitável mesmo em cenários ótimos de redução de emissões. Sendo essa a posição da AOSIS, que configura um dos grupos dos países mais interessados na questão da migração ambiental, é pouco provável que surja qualquer avanço na proteção ao migrante ambiental dentro do regime internacional de mudança climática; ao menos enquanto não houver alteração significativa nos resultados relativos à mitigação. Isso se explica não pelo poder estrutural da Aliança - que não é capaz de grande influência direta em resultados das negociações internacionais -, mas pelo assunto não ser de interesse de atores relevantes, como Estados Unidos e China. Enquanto a AOSIS for o principal ator a trazer o tema para o debate das mudanças climáticas, sua atuação é capaz de orientar a ação dos demais países através da boa aplicação de estratégias de liderança, especialmente a moral, como já foi feito anteriormente em relação a propostas de mitigação.

Considerando, porém, que a migração ambiental está na confluência de diferentes regimes e campos jurídicos, ainda há a possibilidade de promover soluções para a questão sob outras perspectivas teóricas e políticas, especialmente na interface entre mudança climática e direitos humanos, colocando o migrante no centro de qualquer esforço político (CORENDEA; BELLO; BRYAR, 2015; CORENDEA, 2016). As contribuições efetivas que o regime internacional de mudança climática pode oferecer às problemáticas da migração ambiental, entretanto, provavelmente ficarão reduzidas à identificação do fenômeno migratório decorrente da intervenção antrópica em sistemas naturais.

Um breve olhar para a atual conjuntura contribui para colocar mais dúvidas sobre a capacidade do regime internacional de mudança climática de apresentar soluções para a migração ambiental 
sob perspectiva global. O recrudescimento de políticas que advogam a soberania nacional em detrimento de causas transnacionais como a mudança climática e a migração (por exemplo, em Estados Unidos, Brasil, Israel e Hungria, que chegaram a se retirar de acordos relacionados a ambos os temas) pode enfraquecer o regime e minar sua efetividade, atrasando ainda mais a adoção de tratados internacionais sobre migração ambiental.

\section{Referências}

ADGER, William Neil et al. Cultural dimensions of climate change impacts and adaptation. Nature Climate Change, [S. l.], v. 3, p. 112-117, 2013. https://doi.org/10.1038/nclimate1666

AOSIS. Alliance of Small Island States Leaders' Declaration. 2012a. Disponível em: http://aosis.org/ documents/climate-change. Acesso em: 02 de outubro de 2018.

AOSIS. Statement by Nauru on behalf of the Alliance of Small Island States. Ad Hoc Working Group on the Durban Platform for Enhanced Action Opening Plenary. 2012b. Disponível em: http://aosis. org/documents/climate-change. Acesso em: 02 de outubro de 2018.

AOSIS. Statement by Nauru on behalf of the Alliance of Small Island States at the opening of the Ad Hoc Working Group on the Durban Platform for Enhanced Action. 2014. Disponível em: https:// unfccc.int/files/documentation/submissions_from_parties/adp/ application/pdf/adp2.4_aosis_20140310.pdf. Acesso em: 02 de outubro de 2018.

AOSIS. Statement delivered by Nauru on behalf of the Alliance of Small Island States (AOSIS). Closing Plenary Ad-Hoc Working Group on the Durban Platform for Enhanced Action (ADP). 2012C. Disponível em: http://aosis.org/documents/climate-change. Acesso em: 02 de outubro de 2018.

BERTRAM, Geoff. The MIRAB model twelve years on. The Contemporary Pacific, v. 11, n. 1, p. 105$138,1999$.

BETZOLD, Carola. 'Borowing' power to influence international negotiations: AOSIS in the Climate Change Regime, 1990-1997. Politics, [S. l.], v. 30, n. 3, p. 131-148, 2010. https://doi.org/10.1111/j. $1467-9256.2010 .01377 . x$

BETZOLD, Carola; CASTRO, Paula; WEILER, Florian. AOSIS in the UNFCCC negotiations: from unity to fragmentation? Climate Policy, London, v. 12, n. 5, p. 591-613, 2012. https://doi.org/10.1080/14 $\underline{693062.2012 .692205}$

BHANDARY, Rishikesh Ram. Coalition strategies in the climate negotiations: an analysis of mountain-related coalitions. International Environmental Agreements, [S. l.], v. 17, p. 173-190, 2017. https://doi.org/10.1007/s10784-015-9313-6

BIESBROEK, Robbert; SWART, Rob; VAN DER KNAAP, Wim. The mitigation-adaptation dichotomy and the role of spatial planning. Habitat International, Oxford, v. 33, n. 3, p. 230-237, 2009. https:// doi.org/10.1016/j.habitatint.2008.10.001

BOSELLO, Francesco; CARRARO, Carlo; DE CIAN, Enrica. Climate policy and the optimal balance between mitigation, adaptation and unavoided damage. Climate Change Economics, [S. I.], v. 1, n. 2, p. 71-92, 2010. https://doi.org/10.1142/S201000781000008X

CORENDEA, Cosmin. Hybrid legal approaches towards climate change: concepts, mechanisms and implementation. Annual Survey of International \& Comparative Law, [S. l.], v. 21, n. 1, p. 29-41, 2016. 
CORENDEA, Cosmin; BELLO, Valeria; BRYAR, Timothy. Promoting human security and minimizing conflict associated with forced migration in the Pacific region. Barcelona: UNU-GCM, 2015.

CORENDEA, Cosmin; MANI, Tanvi. The progression of climate change, human rights, and human mobility in the context of transformative resilience - a perspective over the Pacific. In: Zinta Zommers; Keith Alverson (org.). Resilience: the science of adaptation to climate change. Amsterdam: Elsevier, 2018. p. 305-316. https://doi.org/10.1016/B978-0-12-811891-7.00025-6

DE ÁGUEDA CORNELOUP, Inés; MOL, Arthur. Small island developing states and international climate change negotiations: the power of moral «leadership». International Environmental Agreements, [S. l.], v. 14, p. 281-297, 2014. https://doi.org/10.1007/s10784-013-9227-0

FALKNER, Robert. The Paris Agreement and the new logic of international climate politics. International Affairs, [S. l.], v. 92, n. 5, p. 1107-1125, 2016. https://doi.org/10.1111/1468-2346.12708

FARBOTKO, Carol; LAZRUS, Heather. The first climate refugees? Contesting global narratives of climate change in Tuvalu. Global Environmental Change, Guildford, v. 22, p. 382-390, 2012. https:// doi.org/10.1016/j.gloenvcha.2011.11.014

GEMENNE, François. Why the numbers don't add up: a review of estimates and predictions of people displaced by environmental changes. Global Environmental Change, Guildford v. 21, p. S41-S49, 2011. https://doi.org/10.1016/j.gloenvcha.2011.09.005

GLOBAL Compact for Safe, Orderly and Regular Migration. 13 de julho de 2018. [S. l. : s. n.], 2018. Disponível em: https://refugeesmigrants.un.org/sites/default/files/180713_agreed_outcome_global_compact_for_migration.pdf. Acesso em: 29 de janeiro de 2019.

HOEGH-GULDBERG, Ove et al. Impacts of $1.5^{\circ} \mathrm{C}$ global warming on natural and human systems. In: Valerie Masson-Delmotte et al. (org.). Global warming of $1.5^{\circ} \mathrm{C}$ : An IPCC Special Report on the impacts of global warming of $1.5^{\circ} \mathrm{C}$ above pre-industrial levels and related global greenhouse gas emission pathways, in the context of strengthening the global response to the threat of climate change, sustainable development, and efforts to eradicate poverty. 2018. cap. 3, p. 175-311.

KAVALIKU, Langi. Culture and sustainable development in the Pacific. In: Anthony Hooper (org.). Culture and sustainable development in the Pacific. Canberra: ANU Press, 2005. p. 22-31.

KELMAN, Ilan. No change from climate change: vulnerability and small island developing states. The Geographical Journal, [S. l.], v. 180, n. 2, p. 120-129, 2014. https://doi.org/10.1111/geoj.12019

KLEIN, Naomi. Foi o capitalismo, e não a «natureza humana», que matou nossos esforços contra as mudanças climáticas. The Intercept . New York, 10 de ago. 2018. Disponível em: https://theintercept. com/2018/08/03/climate-change-new-york-times-magazine. Acesso em: 20 de janeiro de 2019.

KOLMANNSKOG, Vikram. Climate change, environmental displacement and international law. Journal of International Development, [S. l.], v. 24, n. 8, p. 1071-1081, 2012. https://doi.org/10.1002/jid.2888

LARSEN, Henrik. Foreign policy and discourse analysis: France, Britain and Europe. Padstow: Routledge, 1997.

LEE, Donna; HAMPTON, Mark; JEYACHEYA, Julia. The political economy of precarious work in the tourism industry in small island developing states. Review of International Political Economy, [S. l.], v. 22, n. 1, p. 194-223, 2015. https://doi.org/10.1080/09692290.2014.887590

MCNAMARA, Karen Elizabeth; GIBSON, Chris. 'We do not want to leave our land': Pacific ambassadors at the United Nations resist the category of 'climate refugees'. Geoforum, [S. l.], v. 40, p. 475-483, 2009. https://doi.org/10.1016/j.geoforum.2009.03.006 
MINISTRY OF ECONOMY OF REPUBLIC OF FIJI. Planned relocation guidelines: a framework to undertake climate change related relocation. 2018. Disponível em: https://cop23.com.fj/wp-content/ uploads/2018/12/CC-PRG-BOOKLET-22-1.pdf. Acesso em 29 de janeiro de 2019.

MORTREUX, Colette; BARNETT, Jon. Climate change, migration and adaptation in Funafuti, Tuvalu. Global Environmental Change, Guildford, v. 19, p. 105-112, 2009. https://doi.org/10.1016/j.gloenvcha.2008.09.006

MYERS, Norman. Environmental refugees: a growing phenomenon of the 21st century. Philosophical Transactions of the Royal Society of London. Series B: Biological Sciences, London, v. 357, n. 1420, p. 609-613, 2002. https://doi.org/10.1098/rstb.2001.0953

OIM. Glossário sobre migração. Genebra: Organização Internacional para as Migrações, 2009. Disponível em: http://publications.iom.int/system/files/pdf/iml22.pdf>. Acesso em: 28 de janeiro de 2019.

ONU. Brasil: ONU diz que é "lamentável” saída de um Estado-membro do Pacto para Migração. Onu News, [S. l.], og de janeiro de 2019. Disponível em: https://news.un.org/pt/story/2019/01/1654722>. Acesso em: 29 de janeiro de 2019.

ROGELJ, Joeri et al. Paris Agreement climate proposals need a boost to keep warming well below $2{ }^{\circ} \mathrm{C}$. Nature, London, v. 534, p. 631-639, 2016. https://doi.org/10.1038/nature18307

SKODVIN, Tora; ANDRESEN, Steinar. Leadership revisited. Global Environmental Politics, [S. l.], v. 6, n. 3, p. 13-27, 2006. https://doi.org/10.1162/glep.2006.6.3.13

THOMAS, Adelle; BENJAMIN, Lisa. Policies and mechanisms to address climate-induced migration and displacement in Pacific and Caribbean small island developing states. https://doi.org/10.1108/ IJCCSM-03-2017-0055

International Journal of Climate Change Strategies and Management, [S. l.], v. 10, n. 1, p. 86-104, 2018. TOL, Richard. Adaptation and mitigation: trade-offs in substance and methods. Environmental Science \& Policy, Exeter, v.8, n.6, p. 572-578, 2005. https://doi.org/10.1016/j.envsci.2005.06.011 WONG, Poh Poh. Small island developing states. WIREs Climate Change, [S. l.], v. 2, n. 1, p. 1-6, 2011. https://doi.org/10.1002/wcc.84

YOUNG, Oran. Political leadership and regime formation: on the development of institutions in international society. International Organization, Cambridge, Mass., v. 45, n.3, p. 281-308, 1991. https://doi.org/10.1017/So020818300033117

Recebido: 10/04/2019

Aceito: 02/05/2019

\section{Biografia do autor}

\section{Thales Jéferson Rodrigues Schimitt}

Graduando em Relações Internacionais da Universidade Federal do Rio Grande do Sul (UFRGS), bolsista de Iniciação Científica voluntária do Grupo de pesquisa sobre Refugiados, Imigrantes e Geopolítica (GRIGs). ORCID : https://orcid.org/0000-0002-7951-6979; E-mail: thalesjrschimitt@gmail.com 


\section{Verônica Korber Gonçalves}

Professora do Curso de Graduação em Relações Internacionais e do Curso de Pós-Graduação em Estudos Estratégicos Internacionais da Universidade Federal do Rio Grande do Sul (UFRGS). Doutora em Relações Internacionais pela Universidade de Brasília (UNB). Mestre em Sociologia Política pela Universidade Federal de Santa Catarina (UFSC). Graduada em Direito e Mestre em Direito pela Universidade Federal de Santa Catarina.ORCID : https://orcid.org/0000-0001-7144-4707 ; E-mail: veronica.goncalves@ufrgs.br

Instituição: universidade Federal do Rio Grande do Sul (UFRGS).

Localização: Av. Paulo Gama, 110 - Farroupilha, Porto Alegre - RS, 90040-060 